\title{
Advancing the Hexapole Cs-Corrector for the Transmission Electron Microscope
}

Thomas Riedel, Peter Hartel, Martin Linck, Volker Gerheim, Heiko Müller and Stephan Uhlemann

CEOS GmbH, Heidelberg, Baden-Wurttemberg, Germany

After 1998, the correction of image aberrations in TEM [1] has been a huge success. Compared to its first commercial version the basic design of the TEM corrector (CETCOR) has hardly changed over the years. However, recent instrumental developments (mechanical stability, cold FEGs, monochromators, low-kV applications) have pushed the requirements on aberration correctors to a new level. In order to enable high-resolution phase contrast imaging up to the improved information limit of the TEM, it is mandatory to correct for the dominating six-fold astigmatism A5 and to enable full fourth-order axial aberration correction; this is out of reach with the present CETCOR design. Fig. 1a illustrates the impact of A5 for phase contrast imaging at $80 \mathrm{kV}$. Additionally, with further improvements of the microscope resolution the intrinsic off-axial star aberration $\mathrm{S} 3 \mathrm{~g}$ and off-axial three-fold astigmatism $\mathrm{A} 3 \mathrm{~g}$ significantly limit the available field of view for imaging. Last but not least, parasitic off-axial aberrations such as off-axial twofold astigmatism and two-fold anisotropic off-axial coma B2 $g$ often deteriorate the image quality within the recorded field of view. A careful redesign of the CETCOR with optimized hexapole geometries and additional stigmators can remove these limitations for aberration-corrected TEM.

Already several years ago, the hexapole corrector for STEM (CESCOR) had undergone a substantial facelift introducing length-optimized hexapoles for A5-minimization and new degrees of freedom for full fourth-order axial aberration correction [2]. This corrector (DCOR/ASCOR) was well-received in the aberration-corrected STEM community [e.g. 3]. In 2014, CEOS transferred this technology for the first time to an image aberration corrector for a $1.2 \mathrm{MeV}$ TEM within the FIRST project [4]. For correction of the unavoidable azimuthal off-axial coma, the BCOR had been designed [5]. At the same time, the BCOR introduced several additional degrees of freedom to correct for low-order parasitic off-axial aberrations. This knowledge has substantially influenced the redesign of the "old" CETCOR.

A prototype of the advanced TEM corrector has successfully been put into operation in a JEOL JEMARM200F NEOARM electron microscope equipped with high-resolution pole-piece and cold FEG. Since the dimensions and interfaces of the corrector have not changed, it easily replaces the previous CETCOR in the microscope column. According to Fig. 2a the corrector inherits the short monolithic DCOR-type hexapoles which include quadrupole channels. Consequently, the corrector has a minimized A5 and offers full fourth-order axial aberration correction. Furthermore, the intrinsic off-axial aberrations S3g and A3g are strongly reduced and hence improve the available field of view. As additional benefit, three-fold distortions in diffraction patterns are strongly reduced (Fig. 1b). Moreover, the BCOR-type flex pcb coil technology within the transfer lens doublets (two additional hexapoles and quadrupoles, respectively) and additional stigmators above and below the corrector have been incorporated into the new corrector. These measures allow to completely remove the small residual A5 (Fig. 2b) and to independently correct for the two types of parasitic off-axial astigmatism and the off-axial defocus (tilt of image plane) as well as the parasitic two-fold off-axial coma B2g.

We acknowledge and highly appreciate the collaboration and support by JEOL company. [6] 

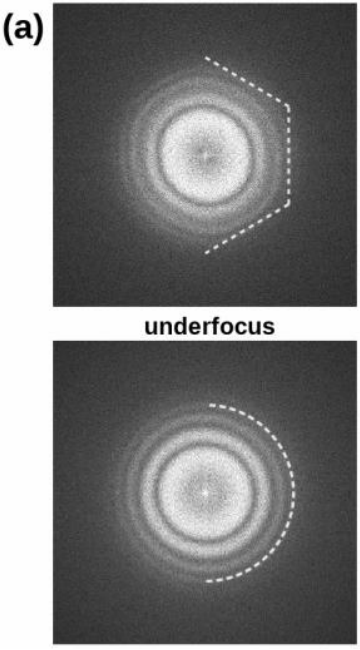

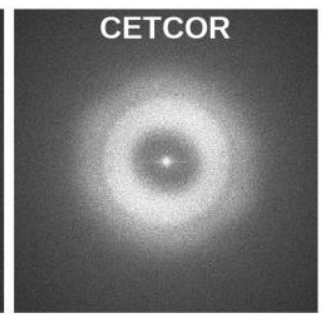

phase contrast

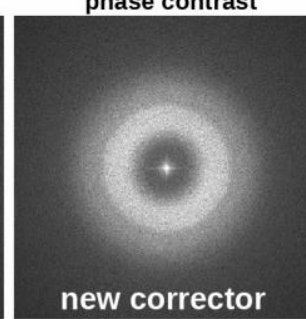

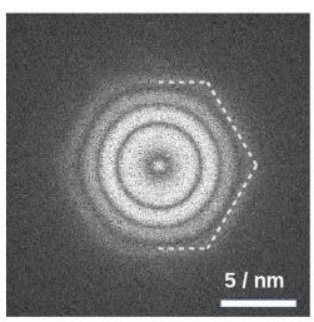

overfocus

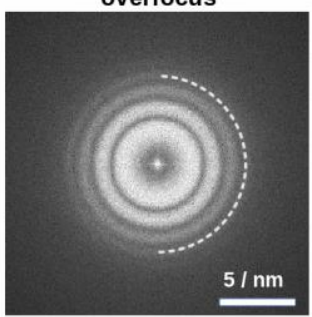

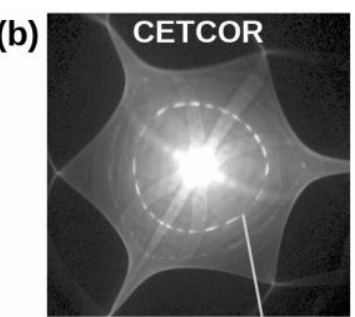

CBED of Si 112

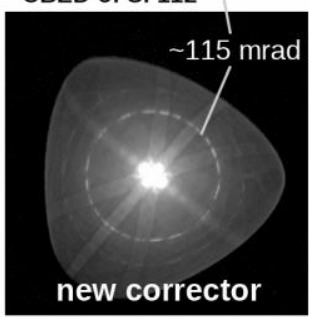

Figure 1. (a) Impact of the six-fold astigmatism A5 on the phase contrast transfer function: The diffractograms with and without A5 have been recorded in a JEOL JEM-ARM200F NEOARM electron microscope equipped with a CETCOR (top) and the advanced TEM corrector (bottom), respectively. (b) Diffraction patterns (CBED) of Silicon in 112-orientation: The huge three-fold and six-fold distortions present with the CETCOR can be strongly reduced with the new corrector.

(a)

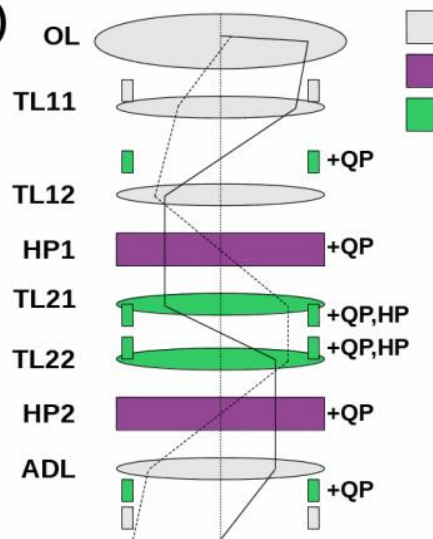

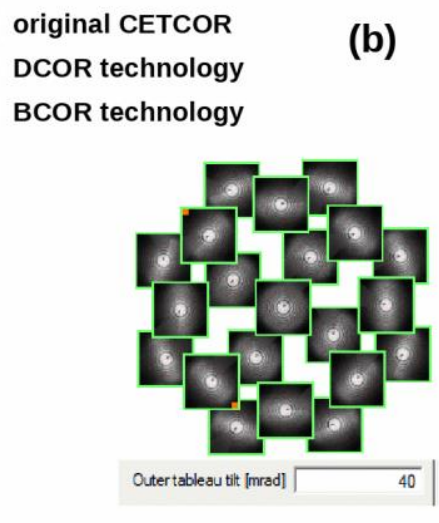

Measurement results:
\begin{tabular}{|l|l|l|l|}
\hline \multicolumn{1}{|c|}{ Value } & Angle & Confidence \\
\cline { 2 - 4 } C1 & $-290 \mathrm{~nm}$ & - & $1.53 \mathrm{~nm}$ \\
\hline A1 & $1.6 \mathrm{~nm}$ & $-143.9^{\circ}$ & $1.24 \mathrm{~nm}$ \\
\hline A2 & $29.2 \mathrm{~nm}$ & $-21.1^{\circ}$ & $27.6 \mathrm{~nm}$ \\
\hline B2 & $2.41 \mathrm{~nm}$ & $-14.3^{\circ}$ & $24.1 \mathrm{~nm}$ \\
\hline C3 & $-9.89 \mu \mathrm{m}$ & - & $1.89 \mu \mathrm{m}$ \\
\hline A3 & $199 \mathrm{~nm}$ & $-6.4^{\circ}$ & $249 \mathrm{~nm}$ \\
\hline S3 & $108 \mathrm{~nm}$ & $-20.8^{\circ}$ & $146 \mathrm{~nm}$ \\
\hline A4 & $2.54 \mu \mathrm{m}$ & $-56.1^{\circ}$ & $4.97 \mu \mathrm{m}$ \\
\hline D4 & $721 \mathrm{~nm}$ & $168^{\circ}$ & $3.05 \mu \mathrm{m}$ \\
\hline B4 & $3.57 \mu \mathrm{m}$ & $-12.6^{\circ}$ & $5.78 \mu \mathrm{m}$ \\
\hline C5 & $5.01 \mathrm{~mm}$ & - & $670 \mu \mathrm{m}$ \\
\hline A5 & $52.6 \mu \mathrm{m}$ & $-123.8^{\circ}$ & $103 \mu \mathrm{m}$ \\
\hline
\end{tabular}

Figure 2. (a) Outline of the new corrector: The original CETCOR (gray) is complemented by DCOR-type multipoles (magenta) and the BCOR-type transfer lens assembly introducing hexpoles and quadrupoles between the second transfer lens doublet, and additional quadrupoles above and below the corrector (green). This new TEM corrector has the same dimensions as the original CETCOR. (b) The screenshot of a tilt tableau result shows the minimization of A5 and the correction of axial fourth-order aberrations during work in progress. The intrinsic positive fifth-order spherical aberration coefficient C5 is favorable for phase contrast transfer.

\section{References}

[1] M. Haider et al., Nature, 392 (1998) 768-769.

[2] H. Müller et al., Microsc. Microanal. 12 (2006) 442-455.

[3] Watanabe et al., Microsc. Microanal. 22 S3 (2016) 310-311.

[4] M. Haider and H. Müller, Hitachi Review Vol. 65, No. 7 (2016) 28-33.

[5] H. Müller et al., Nucl. Instrum. Methods Phys. Res. A 645 (2011) 20-27.

[6] CEOS GmbH has received funding from the European Union's Horizon 2020 research and innovation program under grant agreement No. 823717 - ESTEEM3. 